\title{
Fine Structure of the Spore Sheath of Some Streptomyces Species
}

\author{
By S. T. WILLIAMS AND R. M. BRADSHAW \\ Department of Botany, University of Liverpool, Liverpool, L69 $3 B X$ \\ J. W. COSTERTON AND A. FORGE \\ Department of Biology, University of Calgary, Calgary, Alberta, Canada
}

(Accepted for publication I7 April 1972)

\begin{abstract}
SUMMARY
The fine structure of the spore sheaths of some Streptomyces species was studied by carbon replication of intact and freeze-etched specimens and by negative staining. Young vegetative hyphae lacked a sheath but developed one before the onset of sporing. Substructures were observed on all sheath material; these differed between species and in sporogenous hyphae at different stages of development. Four components were recognized: hollow or grooved elements; amorphous material; fine fibrillar elements; and subunits of spines and hairs. The interrelationships of the components and the possible roles of the sheath were discussed.
\end{abstract}

\section{INTRODUCTION}

Streptomyces spore chains are enclosed during their development by a thin sheath. Spores of some species appear to be ornamented when examined by electron microscopy. The ornaments, which may be warts, spines, hairs (Küster, 1955) or knobs (Lyons \& Pridham, I97I), do not arise from the spore wall but develop from the sheath as spore delimitation occurs (Arai \& Kuroda, I962; Rancourt \& Lechevalier, I964). Vernon (I955) observed that small 'rodlets' were also present on the sheath of some species and similar structures have since been detected in Streptomyces coelicolor (Hopwood \& Glauert, 196I ; Wildermuth, 1970; Wildermuth, Wehrli \& Horne, 1971), S. hygroscopicus var. angustmyceticus (Dietz \& Mathews, I962), S. venezuelae (Bradley \& Ritzi, 1968; Enquist \& Bradley, 197I) and $S$. viridochromogenes and $S$. glaucescens (Wildermuth, I972a, $b$ ).

When spores are detached from their chains, the sheath may remain partially covering them or they may become free from it (Williams \& Sharples, 1970). Properties of spores, such as their hydrophobicity, are often assigned to the 'spore surface' and the contribution of the sheath is obscure. More information about the structure and composition of the sheath is therefore required. The results from a survey of sheath structure in several species are reported here.

\section{METHODS}

Cultures. The following strains were used: Streptomyces finlayi (hairy sheath), S. glaucescens (hairy), S. griseus (smooth), S. thermoflavus (knobbly), S. venezuelae (smooth), $S$. viridochromogenes (spiny) and Streptomyces sp. (FI) (smooth). They were grown on oatmeal agar (Waksman, $196 \mathrm{r}$ ) at $25^{\circ} \mathrm{C}$ for 2 to 3 weeks, with the exception of $S$. thermoflavus which was grown at $45^{\circ} \mathrm{C}$ for 3 days. In studies of germinating spores, spore imprints were made on sterile coverslips which were then incubated in moist sterile sand for 24 to $48 \mathrm{~h}$. 
Carbon replicas. The procedure used was based on those of Bradley \& Williams (I957) and Dietz \& Mathews (1962). Spores from plate cultures were picked up on clean coverslips which were then coated under vacuum with carbon. Coverslip cultures were similarly treated after air-drying. Squares $(2 \mathrm{~mm})$ of the carbon film were cut and the coverslip dipped into hydrofluoric acid for 2 to $3 \mathrm{~s}$; the squares of film were then floated off in distilled water. After digestion overnight in 5 or $10 \mathrm{~N}$-sodium hydroxide solution, preparations were washed six times in distilled water, picked up on Formvar-coated grids and shadowed with goldpalladium.

Freeze-etching. Spores and hyphae of Streptomyces griseus and S. viridochromogenes were freeze-etched. Surface growth from sporing cultures was harvested, washed with distilled water and centrifuged. Portions of the resulting pellet were frozen rapidly in Freon 22 and stored in liquid air. Freeze etching was carried out with a Balzars BA 510 apparatus using a sublimation time of 2 min. Replicas of etched surfaces were washed in $70 \%(\mathrm{v} / \mathrm{v})$ sulphuric acid, 10\% (w/v) sodium hypochlorite and distilled water before mounting on Formvarcoated grids.

Negative staining. The procedure used was based on that of Brenner \& Horne (1959). Spores from plate cultures were picked up on Formvar-coated grids and stained with a drop of $\mathrm{I} \%(\mathrm{w} / \mathrm{v})$ phosphotungstic acid (adjusted to $\mathrm{pH} 7 \cdot 0$ with sodium hydroxide) for $\mathrm{IO}$ to $30 \mathrm{~s}$. Suspensions of intact or macerated spores were also studied and in these cases the stain was added to the suspension and a drop of the mixture placed on a coated grid (Bradley, 1967).

Replicas of intact material and negatively stained preparations were examined with an A.E.I. EM6B electron microscope operated at $60 \mathrm{kV}$. Replicas of freeze-etched material were examined with an A.E.I. EM80 I instrument operated at $60 \mathrm{kV}$.

\section{RESULTS}

The spores of Streptomyces griseus were studied in greatest details. Replicas of unetched material showed that the loose sheath around the spores was clearly patterned with elongated structures in groups which appeared to be randomly arranged (Fig. I). When germ tubes emerged from the spores they disrupted the sheath and their surfaces were unpatterned, although some elements cast from the spore sheath lay on and around them (Fig. 2). When sporogenous hyphae were formed, the surface pattern re-appeared and became more distinct as spores were delimited (Fig. 3). In freeze-etched preparations of vegetative hyphae, the relatively thin wall had a smooth outer surface (Fig. 4). The underlying membrane had a granular appearance typical of freeze-etched organisms and no distinct structures were visible in the cytoplasm. The walls of spores were approximately twice as thick as those of the vegetative hyphae and peripheral bodies were frequent in the cytoplasm (Fig. 5, 6). The smooth fracture planes of these bodies suggested that they might be lipid granules. Alternatively they may have been membrane-enclosed bodies. The smooth outer surface of the spore wall was overlaid by the sheath which was composed of elongated interwoven elements, similar to the ' paired rodlets' observed in $S$. coelicolor (Wildermuth et al. 197I). In negatively stained preparations of homogenized spore suspensions, elements removed from the sheath were frequently observed (Fig. 7, 8). While their diameter was reasonably constant (about $20 \mathrm{~nm}$ ), their length was variable $(80$ to $250 \mathrm{~nm}$ ) and fragmentation often occurred. Their staining pattern indicated that they were either hollow cylinders or centrally grooved solid or hollow structures (Fig. 9). The 'paired rodlets' on freeze-etched preparations could therefore be more accurately regarded as single elements with a central depression, possibly caused by collapse during specimen preparation. It was also clear that detached 'rodlets' were 

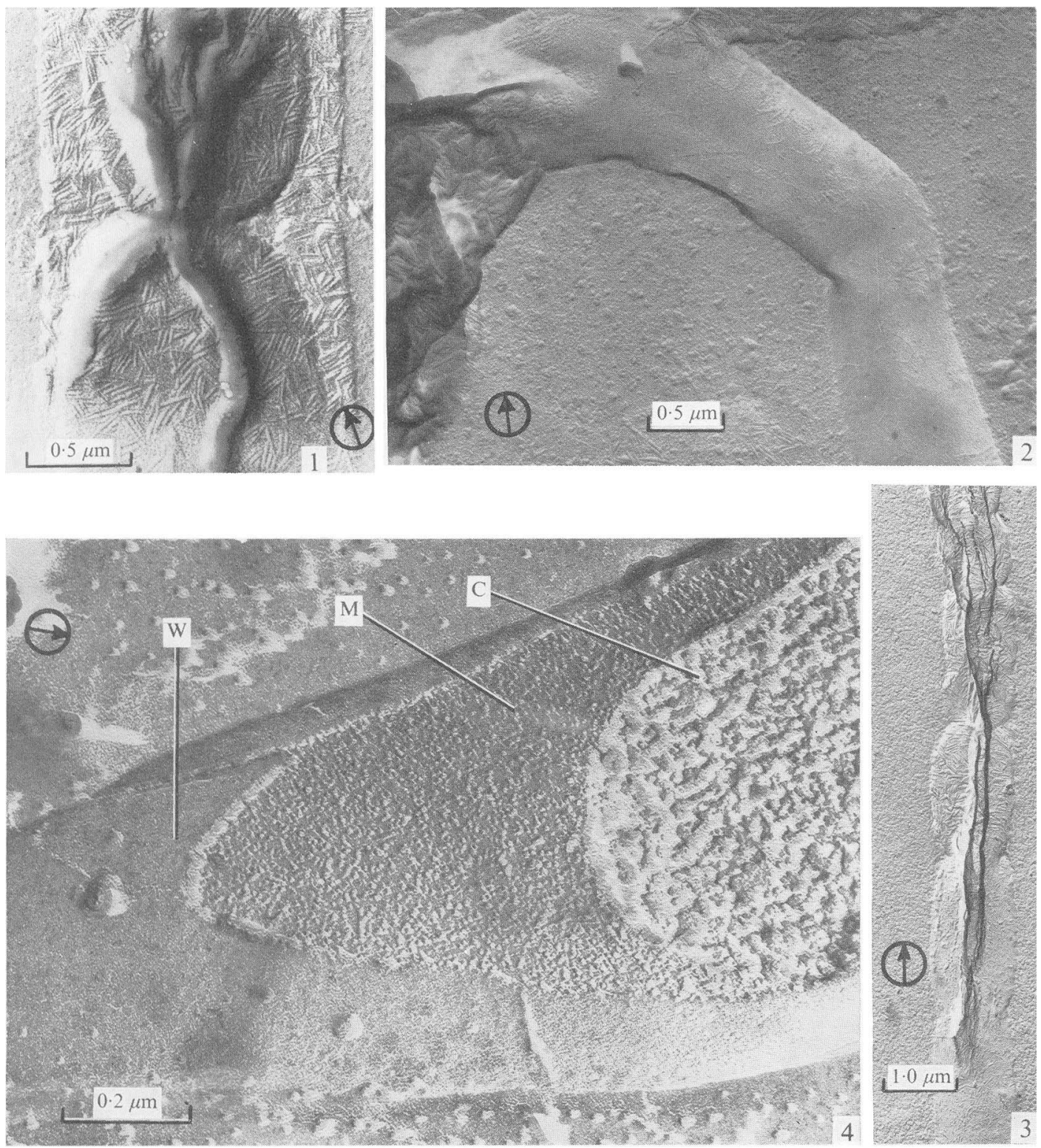

Fig. I. Streptomyces griseus. Carbon replica of sheath overlying spores.

Fig. 2. Streptomyces griseus. Carbon replica of germ tube emerging from spore.

Fig. 3. Streptomyces griseus. Carbon replica of spore chain showing increase in sheath pattern with spore delimitation.

Fig. 4. Streptomyces griseus. Freeze-etched vegetative hypha.

Abbreviations used in Figures: C, cytoplasm; M, membrane; W, wall; PB, peripheral body; $\mathrm{S}$, sheath. 

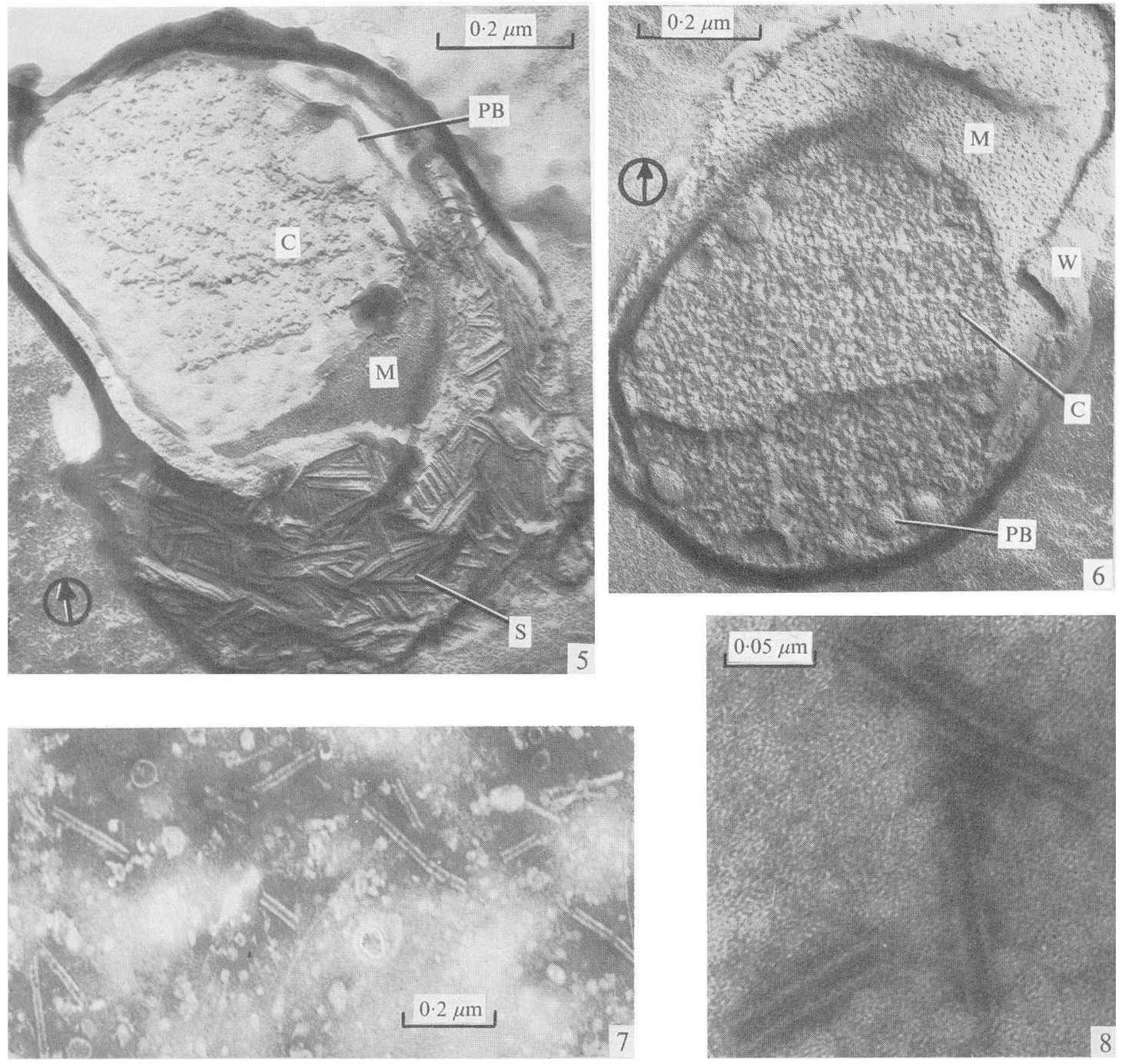

Fig. 5. Streptomyces griseus. Freeze-etched spore.

Fig. 6. Streptomyces griseus. Freeze-etched spore showing peripheral bodies.

Fig. 7. Streptomyces griseus. Negatively stained fragmented sheath elements. Note fragmentation of longer elements.

Fig. 8. Streptomyces griseus. Negatively stained sheath element fragments.

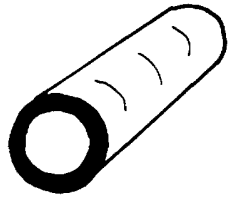

(a)

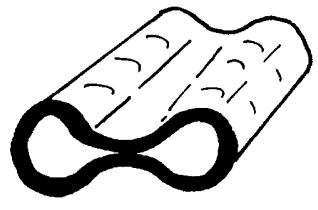

(b)

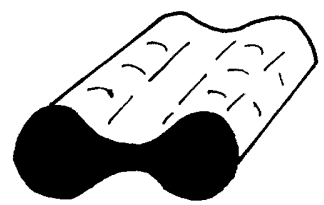

(c)

Fig. 9. Possible structure of sheath elements. (a) Hollow cylindrical; (b) hollow with central depression; $(c)$ solid with central depression. 

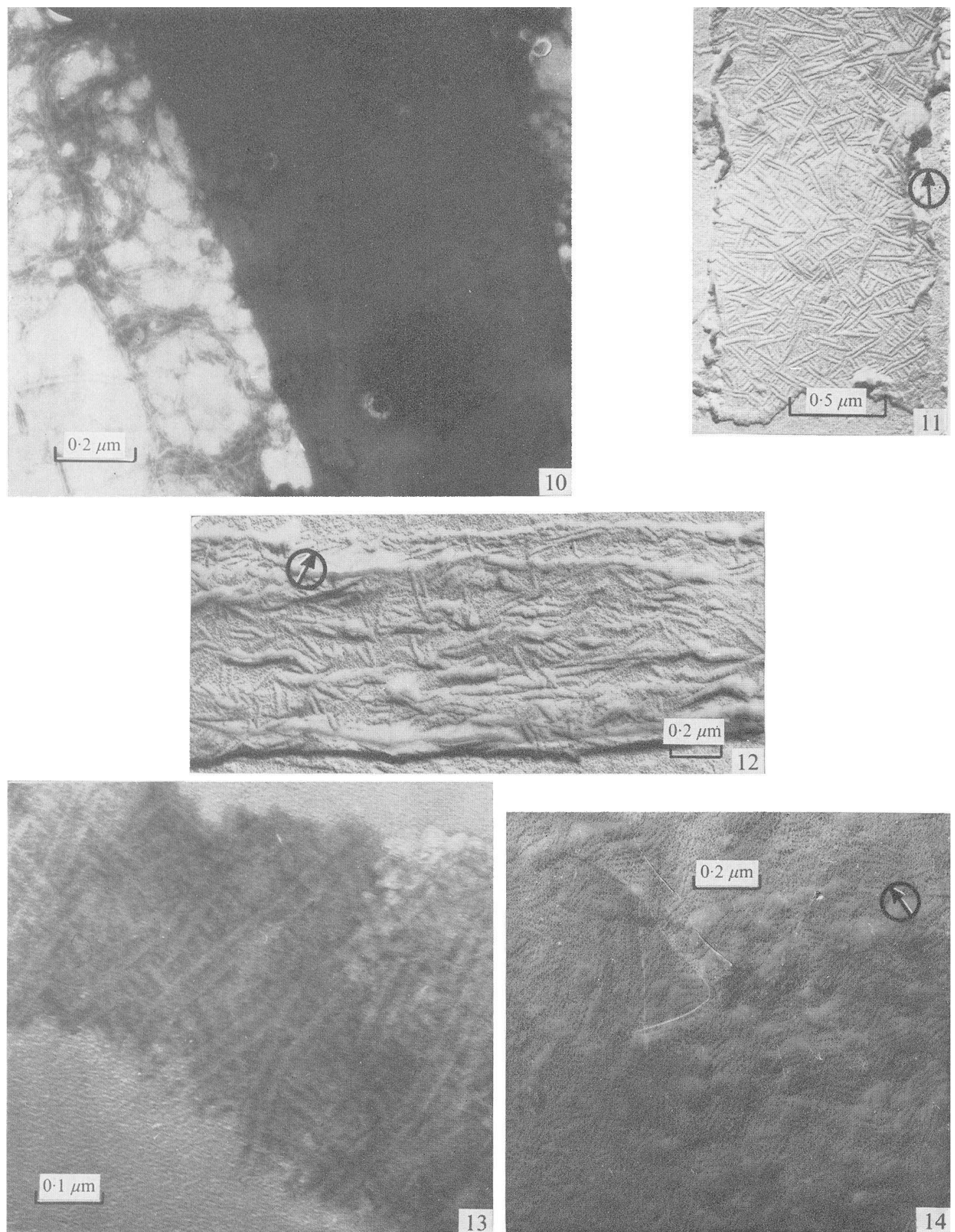

Fig. 10. Streptomyces griseus. Negatively stained thin fibrillar material on non-sporing hypha.

Fig. I I. Streptomyces venezuelae. Carbon replica of sheath overlying spores.

Fig. 12. Streptomyces venezuelae. Carbon replica of young sporogenous hypha.

Fig. 13. Streptomyces venezuelae. Negatively stained piece of sheath.

Fig. I4. Streptomyces sp. (FI). Carbon replica of spore with indistinct pattern. 

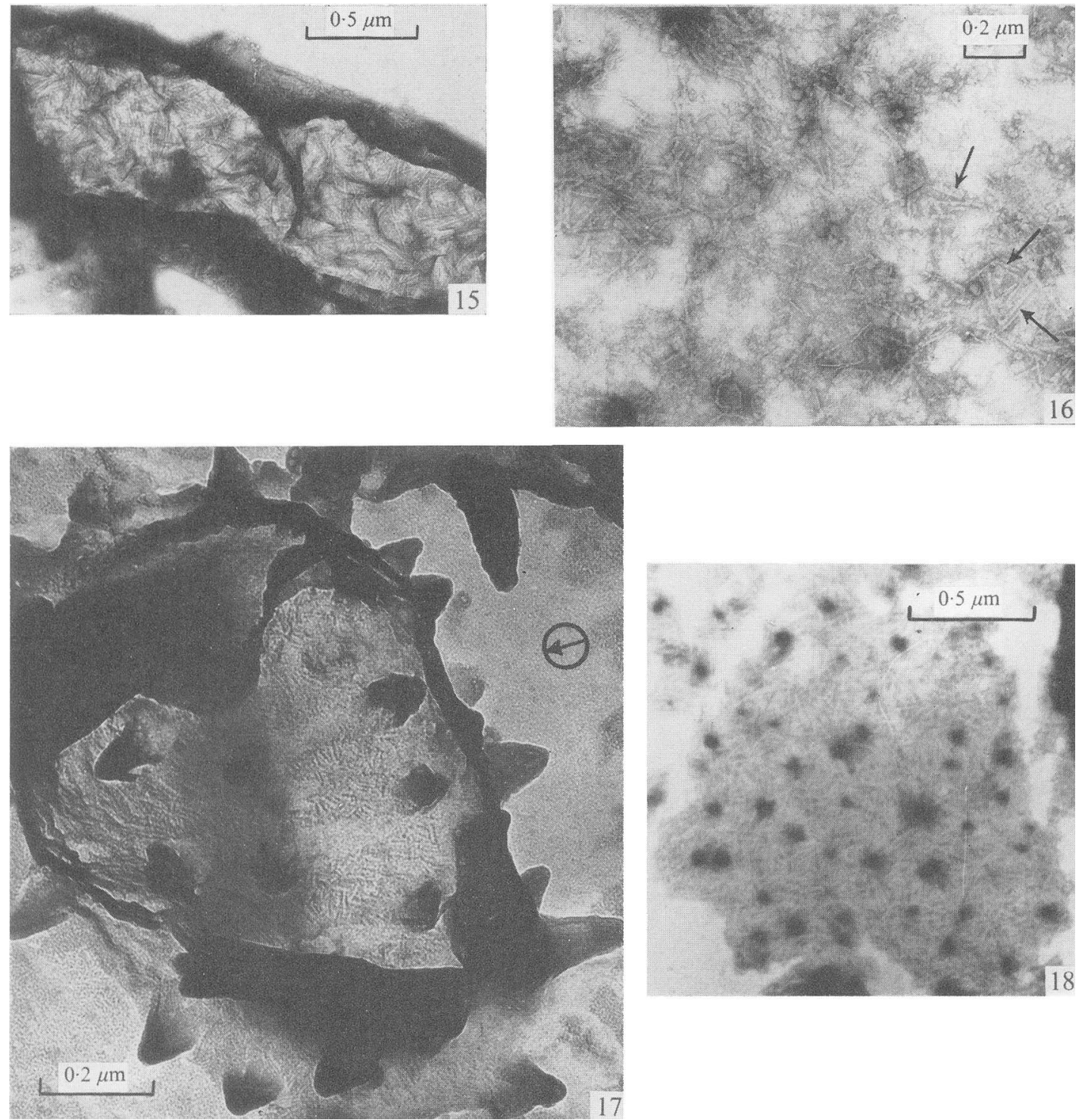

Fig. 15. Streptomyces sp. (F1). Negatively stained spores.

Fig. I6. Streptomyces sp. (FI). Negatively stained fragments from sheath.

Fig. 17. Streptomyces viridochromogenes. Freeze-etched spore with pattern between spines.

Fig. 18. Streptomyces viridochromogenes. Negatively stained piece of sheath.

fragments of elements of undetermined length and should not be regarded as discrete units. Negative staining of non-sporing aerial hyphae revealed fibrillar material which was thinner (about Io $\mathrm{nm}$ ), less clearly stained and less fragmented than the spore sheath elements (Fig. 10).

The sheath around delimited spores of Streptomyces venezuelae was also clearly patterned (Fig. II), and the pattern on hyphae prior to spore delimitation was less distinct (Fig. I2). Negative staining of detached pieces of sheath showed elements which were arranged in at 

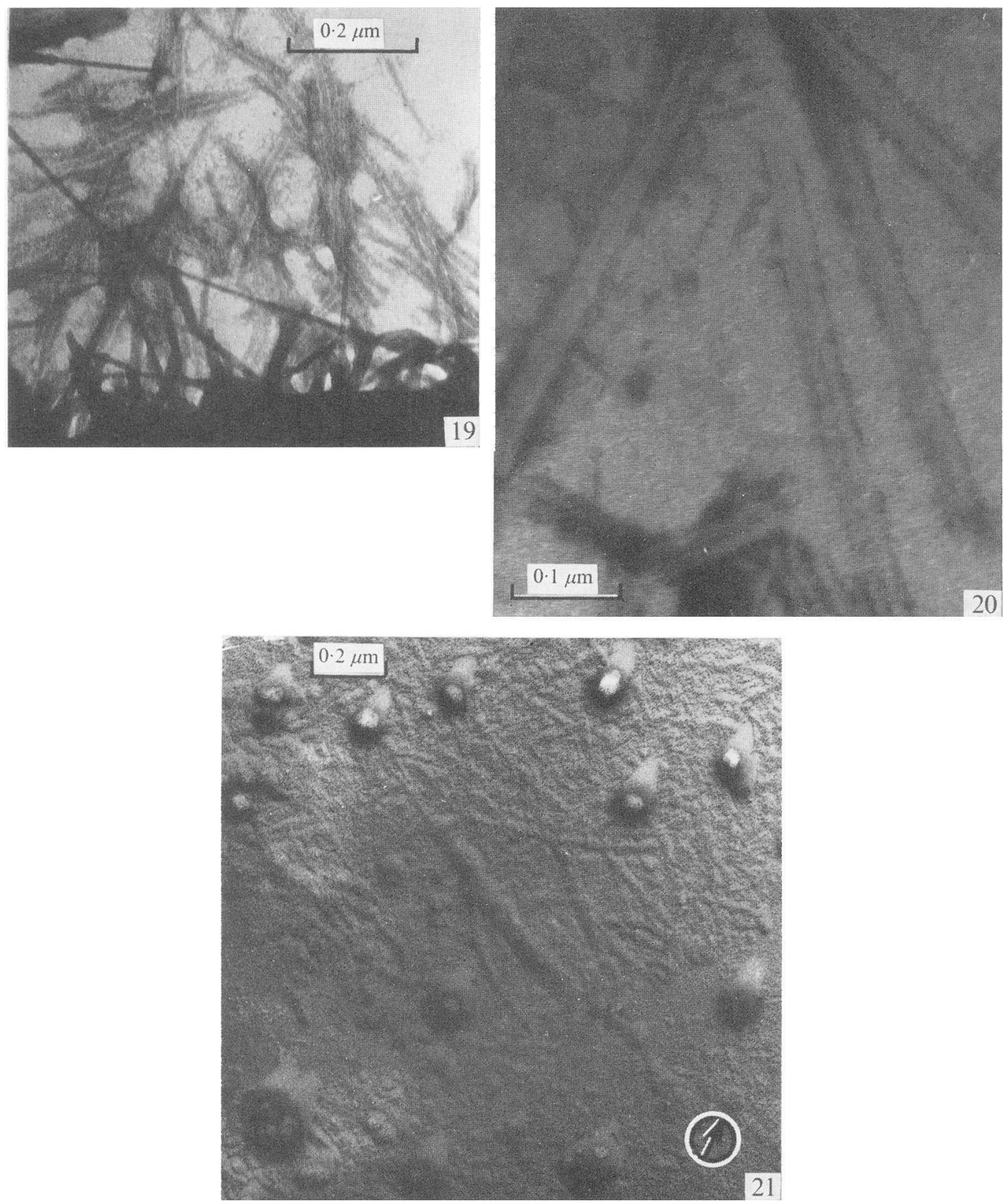

Fig. 19. Streptomyces glaucescens. Negatively stained hair fragments.

Fig. 20. Streptomyces finlayi. Negatively stained hair fragments.

Fig. 21. Streptomyces thermoflavus. Carbon replica of piece of sheath with faint pattern and detached knobs.

least two layers orientated at approximately $90^{\circ}$ to each other (Fig. 13). These elements were structurally similar to those of $S$. griseus but had a larger diameter (about $45 \mathrm{~nm}$ ).

A very indistinct surface pattern was visible on replicas of spores of Streptomyces sp. 
(FI) though it was possible to discern structures similar in size and arrangement to those of Streptomyces griseus (Fig. 14). They appeared to be partially embedded in an amorphous material which obscured their structure. On negatively stained spores, the pattern was more distinct (Fig. 15) and disrupted sheath material consisted mainly of narrow (5 to I5 nm diameter), weakly stained elements of variable length (Fig. I6). These were similar to those on non-sporing hyphae of $S$. griseus (Fig. Io).

On replicas of freeze-etched spores of Streptomyces viridochromogenes a faint pattern was visible on the sheath between the spines (Fig. 17) This consisted of elements approximately I $2 \mathrm{~nm}$ in diameter probably similar to those on the $S$. griseus spore sheath. Some of the spines appeared to be formed by several fold-like units of the sheath. When sheath fragments were negatively stained, a pattern of interwoven elements was observed (Fig. I8). Their staining pattern was not clear but in a few cases a darker central band was present. The radiation of these elements from the sites of the spines suggested that they might contribute to spine formation. It has been clearly demonstrated by Wildermuth (1972a) that spines of this species are made up of 5 to 12 units, each of which consists of a bundle of fibrillar subunits.

Replicas and negatively stained preparations of Streptomyces finlayi and S. glaucescens provided little information about the structure of the sheath between the hairy projections. Wildermuth $(1972 b)$ observed ill-defined rodlet-like elements on the sheath of non-sporing hyphae of the latter species. In negatively stained preparations many hairs remained intact and lacked any visible substructure but when they broke near their bases, many stained fragments were observed (Fig. 19). Staining patterns were similar to those of $S$. griseus spore sheath elements and fragments were of similar diameter $(25 \mathrm{~nm})$ but generally longer. Hair bases appeared to consist of several of these elements. The preparation from $S$. finlayi indicated that these were tubular rather than centrally grooved structures (Fig. 20). These observations and those of Wildermuth $(1972 b)$ indicate that a hair consists of several cylindrical subunits.

Replicas of spores of Streptomyces thermoflavus showed projections from the sheath similar to those described as 'knobs' in S. torulosus (Lyons \& Pridham, 197I) (Fig. 2I). Unlike other projections observed, these often became detached from the sheath. The sheath itself was composed of elongated elements associated with amorphous material which obscured details of their structure.

\section{DISCUSSION}

The results presented here together with those of Wildermuth et al. (I97I) and Wildermuth $(1972 a)$, provide new information on the substructure of the streptomycete sheath. A number of components have been described and it is necessary to consider their relationships.

I. Hollow or centrally grooved elements. These are clearly visible in situ on spore sheaths of Streptomyces griseus, S. venezuelae and S. coelicolor (Wildermuth et al. 197I) and fragments from them ('rodlets') are easily detached. Similar surface patterns have been observed on spores of Aspergillus and Penicillium species (Hess, Sassen \& Remsen, r968; Hess \& Stocks, 1969) and on spore coats of some Bacillus species (Holt \& Leadbetter, 1969; Leadbetter \& Holt, 1969). In other species (e.g. S. viridochromogenes, Streptomyces sp. FI) these elements are less distinct in situ and less readily detached.

2. Amorphous material. This is associated with the above elements and is probably responsible for their lack of clarity and increased stability in some species. It has been described as a 'membrane' on which the elements are held (Wildermuth et al. I97I). 
3. Finer fibrillar elements. These are longer and do not fragment as easily nor stain as clearly as the broader elements. They have been observed on non-sporing aerial hyphae of Streptomyces griseus and $S$. viridochromogenes (Wildermuth, I972a). They may represent a stage in the development of the spore sheath elements.

4. Subunits of spines and hairs. The relationship of these to the basic sheath elements is not yet clear. The basic elements may form these projections but Wildermuth (1972a) has suggested that new material overlying the sheath may be involved.

The sheath of streptomycetes is clearly associated with spore formation. Although absent from primary hyphae, it re-appears on aerial hyphae before spore delimitation begins; development of projections occurs with the onset of sporing (Hopwood \& Glauert, I96I ; Rancourt \& Lechevalier, I964; Bradley \& Ritzi, I968; Williams \& Sharples, 1970). The function of the sheath is not completely understood. It is possible that it protects developing spores and its projections influence the dispersal of spores in soil (Ruddick \& Williams, 1972). Streptomycete spores are usually very hydrophobic, a property which also has an important influence on their dispersal (Ruddick \& Williams, 1972). In a study at present under way, it has been noted that spores of Streptomyces griseus become wettable on removal of their sheath. Therefore hydrophobicity may be brought about by the surface structure of the sheath. It is equally possible that the chemical nature of the sheath is important and an investigation of this is in progress.

We wish to acknowledge a Research Studentship (R.M.B.) and a research grant from the Science Research Council.

\section{REFERENCES}

Arai, T. \& Kur OdA, S. (1962). Fine structure of spiny spores of Streptomyces. Journal of Bacteriology $\mathbf{8 3}$, 924-925.

BradLey, D. E. (1967). Ultrastructure of bacteriophage and bacteriocins. Bacteriological Reviews 31, $230-314$.

Bradley, S. G. \& Ritzi, D. (I968). Composition and ultrastructure of Streptomyces venezuelae. Journal of Bacteriology 95, 2358-2364.

Bradley, D. E. \& Williams, D. J. (1957). An electron microscope study of the spores of some species of the genus Bacillus using carbon replicas. Journal of General Microbiology 17, 75-79.

Brenner, S. \& Horne, R. W. (1959). A negative staining method for high resolution electron microscopy of viruses. Biochimica et biophysica acta 34, 103-I I0.

Dietz, A. \& MATHEws, J. (I962). Taxonomy by carbon replication. I. An examination of Streptomyces hygroscopicus. Applied Microbiology ro, 258-263.

ENQuist, L. W. \& Bradley, S. G. (I97I). Characterisation of deoxyribonucleic acid from Streptomyces venezuelae spores. Developments in Industrial Microbiology 12, 225-236.

Hess, W. M., Sassen, M. M. A. \& Remsen, C. C. (I968). Surface characteristics of Pencillium conidia. Mycologia 60, 290-303.

Hess, W. M. \& Stocks, D. L. (I969). Surface characteristics of Aspergillus conidia. Mycologia 6r, 560-571.

HolT, S. C. \& LeadbetTer, E. R. (I969). Comparative ultrastructure of selected aerobic spore-forming bacteria: a freeze-etching study. Bacteriological Reviews 33, 346-378.

Hopwood, D. A. \& Glauert, A. M. (196I). Electron microscope observations on the surface structures of Streptomyces violaceoruber. Journal of General Microbiology 26, 325-330.

Küster, E. (1955). Beitrag zur Genese und Morphologie der Streptomycetensporen. Zentralblatt für Bakteriologie, Parasitenkunde, Infektionskrankheiten und Hygiene (Abteiltung II) ro8, 376-382.

Leadbetter, E. R. \& Holt, S. C. (I969). Electron microscopy of frozen-etched Bacillus species. In Spores, vol. IV. Edited by L. Leon Campbell. Bethesda, Maryland: American Society for Microbiology.

Lyons, A. J. \& Pridham, T. G. (I97I). Streptomyces torulosus sp. n., an unusual knobbly-spored taxon. Applied Microbiology 22, I90-I93.

Rancourt, M. W. \& Lechevalier, H. A. (1964). Electron microscopic study of the formation of spiny conidia in species of Streptomyces. Canadian Journal of Microbiology ro, 3I I-3I 6. 
RudDick, S. M. \& Williams, S. T. (I972). Studies on the ecology of actinomycetes in soil. V. Some factors influencing the dispersal and adsorption of spores in soil. Soil Biology and Biochemistry 4, 93-103.

VERNON, T. R. (1955). Spore formation in the genus Streptomyces. Nature, London 176, 935-936.

WaKsman, S. A. (1961). The Actinomycetes, vol. 2. Classification, Identification and Description of Genera and Species. Baltimore: Williams \& Wilkins Co.

WILDERMUTH, H. (1970). Surface structure of streptomycetes spores as revealed by negative staining and freeze-etching. Journal of Bacteriology Ior, 318-322.

WiLDERMUTH, H. (1972a). The surface structure of spores and aerial hyphae in Streptomyces viridochromogenes. Archiv für Mikrobiologie 81, 309-320.

WILDERMUTH, H. (1972b). Morphological surface characteristics of Streptomyces glaucescens and S. acrimycini, two streptomycetes with 'hairy' spores. Archiv für Mikrobiologie 81, 32I-332.

WiLDERMUTH, H., WeHRLI, E. \& HoRNE, R. W. (1971). The surface structure of spores and aerial mycelium in Streptomyces coelicolor. Journal of Ultrastructure Research 35, I68-180.

Williams, S. T. \& Sharples, G. P. (1970). A comparative study of spore formation in two Streptomyces species. Microbios 5, 17-26. 